Am. J. Trop. Med. Hyg., 74(5), 2006, pp. 730-732

Copyright () 2006 by The American Society of Tropical Medicine and Hygiene

\title{
ARE PLASMODIUM FALCIPARUM PARASITES PRESENT IN PERIPHERAL BLOOD GENETICALLY THE SAME AS THOSE SEQUESTERED IN THE TISSUES?
}

\author{
EDSON G. DEMBO, HAPPY T. PHIRI, JACQUI MONTGOMERY, MALCOLM E. MOLYNEUX, AND \\ STEPHEN J. ROGERSON* \\ Malawi-Liverpool-Wellcome Trust Clinical Research Programme, Blantyre, Malawi; Department of Medicine, College of Medicine, \\ University of Malawi, Blantyre, Malawi; Liverpool School of Tropical Medicine, Liverpool, United Kingdom; Department of \\ Medicine, University of Melbourne, Royal Melbourne Hospital, Parkville, Victoria, Australia
}

\begin{abstract}
Since quinine does not inhibit the growth of Plasmodium falciparum ring stages or mature schizonts, parasites may continue to emerge from sequestration sites after starting treatment. We used polymerase chain reaction amplification of $P$. falciparum merozoite surface protein 1 (MSP-1) and MSP-2 alleles to distinguish genotypes infecting 58 children with severe malaria. To examine changes in parasite populations in peripheral blood over time, we compared changes in number and spectrum of genotypes in samples on admission to a hospital to those obtained up to 24 hours later. Thirty-four children lost genotypes, 21 retained genotypes, and 3 gained an extra $P$. falciparum genotype at one locus but not the other. The lack of novel genotypes emerging suggests that among children with severe malaria the dominant clones sequestered in deep organs are usually the same as those in peripheral circulation.
\end{abstract}

\section{INTRODUCTION}

Immunity to malaria is acquired slowly in the first decade of life after repeated exposure to Plasmodium falciparum, and young children have the greatest burden of severe disease. Individuals are usually infected with mixtures of genotypes $^{1}$ as a result of sexual reproduction that generates new genotypes ${ }^{2,3}$ and novel alleles by intragenic recombination. ${ }^{4}$

Malaria infections are composed of a variable number of distinct genotypes, with a mean of 2-3 genotypes in one patient sample. In Malawian pregnant women, we have shown that the genotypes present in peripheral blood were frequently different from those in placental blood, suggesting that some genotypes were circulating in the peripheral blood while others were sequestered in the tissues. ${ }^{5}$ We hypothesized that parasites circulating in peripheral blood in children with severe and complicated malaria are genetically distinct from those sequestered in the brain and other organs, and that a proportion of parasites sequestered on admission would emerge into the peripheral blood over an 18-24-hour period post-admission. Data from longitudinal studies in asymptomatic children indicate that such a process occurs in children with asymptomatic malaria infections, with variations in the $P$. falciparum population in blood samples collected on a daily or third-day basis. ${ }^{6,7}$

Among individuals treated with parenteral quinine, parasite clearance time is frequently greater than 24 hours. $^{8}$ If sequestered and circulating parasites are genetically distinct broods, as has been described in asymptomatic infection, ${ }^{7}$ one might expect to find new genotypes emerging in peripheral blood of children under quinine treatment, as a result of continuing schizogony among sequestered parasites and invasion of more erythrocytes by merozoites.

We used a nested polymerase chain reaction (PCR) to amplify genes encoding merozoite surface protein 1 (MSP-1) and MSP-2 to distinguish different genotypes in admission samples and at 18 and/or 24 hours. This method provides the advantage of great sensitivity and specificity, and distinguishes between alleles of similar serologic properties by size

* Address correspondence to Stephen J. Rogerson, Department of Medicine, University of Melbourne, Post Office, Royal Melbourne Hospital, Victoria 3050, Australia. E-mail: sroger@unimelb.edu.au polymorphism. ${ }^{9}$ We asked whether parasites persisting at 18 24 hours after admission had different genotypes to those present on admission, which might indicate different broods of parasites in the periphery and in the tissues.

\section{MATERIALS AND METHODS}

Patient selection and blood sampling. Children admitted to the pediatric ward of the Queen Elizabeth Central Hospital in Blantyre, Malawi between January 2002 and July 2003 with suspected severe and complicated malaria ${ }^{10}$ and parasitemia $\geq 1 \%$ on thin blood film examination were recruited for the study. After consent was obtained from a child's parent or guardian, clinical histories were recorded and the patients were treated with parenteral quinine $(20 \mathrm{mg} / \mathrm{kg}$ loading dose, $10 \mathrm{mg} / \mathrm{kg}$ twice a day). Finger prick blood samples were collected every six hours. Malaria parasitemia was quantitated using Giemsa-stained thick and thin blood films, and $200 \mu \mathrm{L}$ of whole blood was collected into EDTA-containing microtainers for DNA extraction. Samples with microscopically detectable parasitemia at 18 and/or 24 hours were chosen for DNA extraction. All samples were frozen at $-20^{\circ} \mathrm{C}$ until extraction of DNA was performed.

PCR analysis. DNA extraction was performed using the QIAamp DNA Mini Kit (Qiagen, Crawley, United Kingdom). DNA was eluted in $200 \mu \mathrm{L}$ of $10 \mathrm{mM}$ Tris- $\mathrm{HCl}, 0.5 \mathrm{mM}$ EDTA, pH 9.0, and frozen at $-20^{\circ} \mathrm{C}$. Samples were genotyped by a nested PCR using primer pairs specific for amplifying the known allelic families of MSP-1 and MSP-2. ${ }^{11}$ The targeted polymorphic regions of MSP-1 and MSP-2 were amplified in a primary PCR using $1 \mu \mathrm{L}$ of template DNA on a PTC-200 thermal cycler (MJ Research Inc., Watertown, MA) under the following conditions: $94^{\circ} \mathrm{C}$ for 5 minutes, 25 cycles at $94^{\circ} \mathrm{C}$ for 1 minute, $58^{\circ} \mathrm{C}$ for 2 minutes, and $72^{\circ} \mathrm{C}$ for 1 minute, and a final extension at $72^{\circ} \mathrm{C}$ for 2 minutes. All reactions were conducted in a volume of $20 \mu \mathrm{L}$ and contained $125 \mu \mathrm{M}$ dNTPs, $125 \mathrm{nM}$ oligonucleotides, and 0.5 units of Taq polymerase. Two microliters of primary product was used to initiate a secondary reaction with specific nested oligonucleotide pairs targeting each of the allelic families of MSP-1 and MSP2. This reaction used 30 cycles as above with an annealing temperature of $61^{\circ} \mathrm{C}$. Positive and negative controls were included in all batches. 
All products were analyzed by electrophoresis in 2\% agarose gels $(0.5 \times$ Tris-borate-EDTA buffer $)$, stained with ethidium bromide, and visualized by ultraviolet transillumination. Each patient's matched samples were run in adjacent wells in all assays. The number of bands visualized within expected size ranges ${ }^{11}$ were counted for each allelic family and summed. The minimum number of genotypes was specified as the highest total number of bands within either the MSP-1 or MSP-2 allelic families. ${ }^{6}$ All samples that resulted in different bands at 18 or 24 hours from admission samples were retested for confirmation.

Ethical approval. This study was reviewed and approved by the College of Medicine Research and Ethics Committee, University of Malawi.

\section{RESULTS}

During the study period, 295 children with severe and complicated malaria were admitted to the pediatric ward of Queen Elizabeth Central Hospital and 58 children with parasitemia $\geq 1 \%$ completed the study. Reasons for noncompletion included low initial parasitemia, early clearance of parasitemia (before 18 hours), and death of the patient. Twenty-eight children had cerebral malaria alone, 19 had cerebral malaria combined with severe anemia (hematocrit $<15 \%)$, 5 had severe anemia, and 6 had non-severe malaria with or without prostration. Logistic difficulties prevented examination of samples from children who were aparasitemic by microscopy by 18 hours. DNA was extracted and PCR was performed at 0,18 , and 24 hours in 33 patients, at 0 and 24 hours in 18 patients, and 0 and 18 hours in 7 patients. Parasite genotypes studied by PCR amplification of three MSP-1 and two MSP-2 alleles showed a mean of 2-3 genotypes per patient (Table 1).

Comparing the number of genotypes at 0,18 , and 24 hours, we found that 3 patient isolates gained extra genotypes at either MSP-1 or MSP-2 2 loci, 13 patient isolates lost genotypes at either MSP-1 or MSP-2, 21 patient isolates lost genotypes at both MSP-1 and MSP-2, and 21 patient isolates retained all their genotypes throughout the 24-hour period (Table 2).

The single isolate that gained genotypes at the MSP-1 locus gained one genotype at the 24-hour time point in addition to an existing genotype for RO33 and gained two new genotypes for K1. For the two isolates that gained genotypes at the MSP-2 locus only, one isolate gained a new genotype for IC at the 24-hour point (this had no existing genotype for IC) and another isolate gained one FC27 genotype in addition to an

TABLE 1

Average number of genotypes and distribution of merozoite surface protein 1 (MSP 1) and MSP2 alleles relative to time from admission in Malawian children with severe malaria

\begin{tabular}{|c|c|c|c|c|c|c|}
\hline \multirow{3}{*}{$\begin{array}{l}\text { Time after } \\
\text { admission } \\
\text { (hours) }\end{array}$} & \multirow{3}{*}{$\begin{array}{l}\text { Maximum no. } \\
\text { of genotypes } \\
\text { detected* }\end{array}$} & \multicolumn{5}{|c|}{ Frequency of alleles } \\
\hline & & \multicolumn{3}{|c|}{ MSP 1} & \multicolumn{2}{|c|}{ MSP 2} \\
\hline & & MAD20 & KI & RO33 & $\mathrm{FC} 27$ & IC \\
\hline 0 & $3.0 ; 3.0 ;(1-8)$ & $81 \%$ & $53 \%$ & $62 \%$ & $64 \%$ & $90 \%$ \\
\hline 18 & $2.2 ; 2.0 ;(0-6)$ & $73 \%$ & $46 \%$ & $46 \%$ & $49 \%$ & $89 \%$ \\
\hline 24 & $2.1 ; 2.0 ;(0-5)$ & $76 \%$ & $33 \%$ & $41 \%$ & $59 \%$ & $84 \%$ \\
\hline
\end{tabular}

*Values are the mean; median; (range).
TABLE 2

Number of Malawian children with severe and complicated malaria who lost, gained, or retained genotypes*

\begin{tabular}{llcc}
\hline \multicolumn{1}{c}{ Marker(s) } & $\begin{array}{c}\text { Gained } \\
\text { no. }(\%)\end{array}$ & $\begin{array}{c}\text { Lost } \\
\text { no. (\%) }\end{array}$ & $\begin{array}{c}\text { Retained } \\
\text { no. }(\%)\end{array}$ \\
\hline MSP 1 only & $1(1.7)$ & $9(15.5)$ & $\dagger$ \\
MSP 2 only & $2(3.4)$ & $4(6.9)$ & $\dagger$ \\
MSP 1 and MSP 2 & $0(0)$ & $21(36.2)$ & $21(36.2)$ \\
Total & $3(5.2)$ & $34(58.6)$ & $21(36.2)$ \\
\hline
\end{tabular}

* MSP $1=$ merozoite surface protein 1

$\dagger$ All patients who lost MSP 1 genotypes retained their MSP 2 genotypes and those who lost MSP 2 genotypes retained their MSP 1 genotypes. No patient gained genotypes at both loci.

existing genotype. No patient gained genotypes at both loci. There was no association between clinical diagnosis and patterns of change in genotype. Children who died or had sequelae did not have more genotypes on admission than those making a full recovery (median $=3$, range $=1-8$ compared with median $=3$, range $=1-6$ ), but they tended to be more likely to lose genotypes over admission (11 of $34,32 \%$ compared with 2 of $23,9 \%$; $=0.054$, by Fisher's exact test). Outcome was missing on one patient.

\section{DISCUSSION}

Our principal hypothesis was that novel parasite genotypes may emerge into the peripheral blood over the first 24 hours after admission with severe malaria. This phenomenon might be a consequence of differential emergence of different genotypes after schizont rupture. This appears to be the case in asymptomatic malaria, ${ }^{6,7}$ but not in symptomatic infection. ${ }^{12}$ We found no compelling evidence for this in severe disease. Instead, many samples showed a loss of genotypes, consistent with parasite densities decreasing below detection threshold after initiation of therapy (as expected), and a large proportion of patients retained their genotypes over 24 hours despite quinine treatment. Although clearance of parasite DNA from the circulation may be delayed for 24 hours, ${ }^{13}$ and persistent DNA may contribute to our genotype findings, we believe viable parasites are a more likely explanation. All patients had detectable parasitemia by microscopy at each sampling point, and persistence of microscopically identifiable ringstage parasites in peripheral blood well beyond 24 hours was common. In Thai adults, the median time to clearance of parasitemia on quinine treatment was 64 hours. $^{8}$ Persistent genotypes could also be due to gametocytes, which frequently are of mixed genotypes; ${ }^{14}$ we cannot exclude this as a contributing factor.

Rings normally grow to mature trophozoite stage by 18 hours post-invasion, when they disappear from peripheral blood and sequester in tissues. If quinine retarded parasite maturation, rings might persist for 24 hours after starting treatment. However, they appear to be resistant to quinine, ${ }^{15}$ except in the presence of high concentrations that would not be attained within the first 24 hours of treatment, ${ }^{16}$ making this explanation less likely.

There is some evidence that sequestration and schizogony continue for more than 24 hours after initial administration of quinine treatment, ${ }^{8}$ and also that quinine does not significantly reduce cytoadherence. ${ }^{17}$ Thus, repopulation of peripheral blood with progeny of sequestered parasites may con- 
tinue after starting therapy. Our data suggest such repopulation is composed of parasites of the same dominant genotypes as those circulating at admission.

Changes in genotypes at one locus only could be attributed to technical problems. Our nested PCR might have failed to amplify MSP-1 or MSP-2 alleles present at levels below the threshold of sensitivity. Our results show that of 58 patients studied, only $3(5.2 \%)$ had new genotypes detected at either the MSP-1 or MSP-2 loci. If two strains were identical at one locus, a new genotype may show only one new band. Apart from our favored explanation, that sequestered parasites are re-emerging into the circulation, other possible explanations for detection of a single new band also include variations in the quality of DNA or performance of PCR, and competition between different genotypes in our PCRs. By extracting matched samples at the same time, by repeating assays with potential new genotypes, and by running matched samples in adjacent wells in the same assay, we believe we have minimized potential artifacts as the cause of our findings.

Malaria syndrome did not appear to influence the degree of change in genotype seen, although we had relatively few samples from children with severe anemia or uncomplicated malaria. The surprising finding that the proportion of isolates showing genotype loss tended to be higher in children with unfavorable outcomes was not explained either by differences in numbers of genotype on admission, or by differences in proportions of isolates tested at 18 compared with 24 hours, and may warrant further investigation.

The results suggest that dominant clones persist in both circulating parasites and those sequestered in the tissues. These observations confirm and extend those of Missinou and others. ${ }^{12}$ In Gabon, children with single episodes of $P$. falciparum malaria were found to carry parasites with the same genotypes throughout the course of infection. ${ }^{12}$ The observations of genetically distinct, asynchronous broods of parasites in asymptomatic infection ${ }^{6,7}$ do not appear to be relevant to severe malaria pathogenesis, but suggest instead that relatively uniform dominant populations of genotypes may be found in both peripheral blood and tissues. Detailed comparison of peripheral blood and tissues from autopsy studies of children with fatal malaria will resolve this question.

Received May 30, 2005. Accepted for publication December 1, 2005.

Acknowledgments: We thank Professor Terrie Taylor and nursing staff (Research Ward, Department of Paediatrics, College of Medicine, University of Malawi) for collecting samples for the study, and the children and their parents and guardians for their participation.

Financial support: This study was supported by the Wellcome Trust (Senior Fellowship 063215 to Stephen J. Rogerson and project grant to 071376 to Malcolm E. Molyneux).

Authors' addresses: Edson G Dembo, Happy T Phiri, Jacqui Montgomery, and Malcolm E Molyneux, Malawi-Liverpool-Wellcome Trust Clinical Research Programme, Box 30096, Blantyre 3 Malawi, Telephone: 265-167-6444, Fax: 265-167-5774. Stephen J Rogerson, Department of Medicine, Post Office Royal Melbourne Hospital, Parkville, Victoria 3050, Australia, Telephone: 61-3-8344-3259, Fax: 61-3-9347-1863, E-mail: sroger@unimelb.edu.au.

\section{REFERENCES}

1. Daubersies P, Sallenave-Sales S, Magne S, Trape J-P, Contamin $\mathrm{H}$, Fandeur T, Rogier C, Mercereau-Puijalon O, Druilhe P, 1996. Rapid turnover of Plasmodium falciparum populations in asymptomatic individuals living in a high transmission area. Am J Trop Med Hyg 54: 18-26.

2. Walliker D, Quakyi IA, Wellems TE, McCutchan TF, Szarfman A, London WT, Corcoran LM, Burkot TR, Carter R, 1987. Genetic analysis of the human malaria parasite Plasmodium falciparum. Science 236: 1661-1666.

3. Paul RE, Packer MJ, Walmsley M, Lagog M, Ranford-Cartwright LC, Paru R, Day KP, 1995. Mating patterns in malaria parasite populations of Papua New Guinea. Science 269: 1709-1711.

4. Kerr PJ, Ranford-Cartwright LC, Walliker D, 1994. Proof of intragenic recombination in Plasmodium falciparum. Mol Biochem Parasitol 66: 241-248.

5. Kamwendo DD, Dzinjalamala FK, Snounou G, Kanjala MCC, Mhango CG, Molyneux ME, Rogerson SJ, 2002. Plasmodium falciparum: PCR detection and genotyping of isolates from peripheral, placental, and cord blood of pregnant Malawian women and their infants. Trans R Soc Trop Med Hyg 96: 145149.

6. Farnert A, Snounou G, Rooth I, Bjorkman A, 1997. Daily dynamics of Plasmodium falciparum subpopulations in asymptomatic children in a holoendemic area. Am J Trop Med Hyg 56: $538-547$.

7. Bruce MC, Galinski MR, Barnwell JW, Donnelly CA, Walmsley M, Alpers MP, Walliker D, Day KP, 2000. Genetic diversity and dynamics of Plasmodium falciparum and P. vivax populations in multiply infected children with asymptomatic malaria infections in Papua New Guinea. Parasitology 121: 257-272.

8. Davis TM, Supanaranond W, Pukrittayakamee S, Silamut K, White NJ, 2003. Evidence for continued two-brood replication of Plasmodium falciparum in vivo during quinine treatment. Acta Trop 89: 41-45.

9. Zwetyenga J, Rogier C, Tall A, Fontenille D, Snounou G, Trape JF, Mercereau-Puijalon O, 1998. No influence of age on infection complexity and allelic distribution in Plasmodium falciparum infections in Ndiop, a Senegalese village with seasonal, mesoendemic malaria. Am J Trop Med Hyg 59: 726-735.

10. Warrell DA, Molyneux ME, Beales PF, 1990. Severe and complicated malaria. Trans $R$ Soc Trop Med Hyg 84: 1-65.

11. Snounou G, Zhu X, Siripoon N, Jarra W, Thaithong S, Brown KN, Viriyakosol S, 1999. Biased distribution of $m s p 1$ and $m s p 2$ allelic variants in Plasmodium falciparum populations in Thailand. Trans $R$ Soc Trop Med Hyg 93: 369-374.

12. Missinou MA, Kun JF, Kremsner PG, 2004. No change in parasite genotype during single episodes of Plasmodium falciparum malaria in Gabonese children. Parasitol Res 93: 322-327.

13. Jarra W, Snounou G, 1998. Only viable parasites are detected by PCR following clearance of rodent malarial infections by drug treatment or immune responses. Infect Immun 66: 3783-3787.

14. Sutherland C, Alloueche A, McRobert L, Ord R, Leggat J, Snounou G, Pinder M, Targett GA, 2002. Genetic complexity of Plasmodium falciparum gametocytes isolated from the peripheral blood of treated Gambian children. Am J Trop Med Hyg 66: 700-705.

15. Skinner TS, Manning LS, Johnston WA, Davis TM, 1996. In vitro stage-specific sensitivity of Plasmodium falciparum to quinine and artemisinin drugs. Int J Parasitol 26: 519-525.

16. ter Kuile FO, Dolan G, Nosten F, Edstein MD, Luxemburger C, Phaipun L, Chongsuphajaisiddhi T, Webster HK, White NJ, 1993. Halofantrine versus mefloquine in treatment of multidrug-resistant falciparum malaria. Lancet 341: 1044-1049.

17. Udomsangpetch R, Pipitaporn B, Krishna S, Angus B, Pukrittayakamee S, Bates I, Suputtamongkol Y, Kyle DE, White NJ, 1996. Antimalarial drugs reduce cytoadherence and rosetting Plasmodium falciparum. J Infect Dis 173: 691-698. 